\title{
Violência, dependência química e transtornos mentais em presidiários
}

\author{
Violence, drug dependence and mental \\ disturbances in prisoners
}

\author{
Gislaine Pereira TAVARES \\ Rosa Maria Martins de ALMEIDA'
}

\begin{abstract}
Resumo
O presente trabalho realizou uma análise de artigos científicos selecionados nas fontes eletrônicas SciELO, PubMed e Web of Science, no período entre 1998 e 2008. Na elaboração da revisão e na discussão de dados, foram utilizados os descritores: violência (violence); dependência de drogas (drug dependence), transtornos mentais (mental disturbs) e prisioneiros (prisoners). No total, foram encontrados 408 artigos sobre a temática, tendo sido utilizados 60 deles, juntamente com 23 livros e teses que abordam a problemática em questão. A revisão teve por objetivo analisar a relação entre violência, dependência química e transtornos mentais na população carcerária masculina. Os resultados mostraram que o uso abusivo de drogas é um importante fator de risco para a violência e que os transtornos mentais, muitas vezes, acompanham atos violentos. Conclui-se que a violência e a dependência de drogas estão diretamente relacionadas em prisioneiros.
\end{abstract}

Unitermos: Crime. Drogas. Homens. Prisões. Violência.

\begin{abstract}
The present article is an analysis based on scientific articles from internet sources such as SciELO, PUbMed and Web of Science, between 1998 and 2008. For the search, the keywords used were: violence, drug dependence, mental disturbances and prisoners, in order to produce this review and discuss the data. A total of 408 articles were found, of which 60 were considered suitablefor this discussion and 23 books were also found related to this topic. The aim of this study was to analyze the relationship between violence, drug abuse and mental disturbances in male prisoners. The results showed that drug abuse is an important riskfactor in engendering violence and that mental disturbances often go hand in hand with aggressive behavior. It may be concluded that violence and drug dependence in prisoners are directly interrelated.
\end{abstract}

Uniterms: Crimes. Drugs. Men. Prisons. Violence.

A violência é um problema social e de saúde pública que ameaça o desenvolvimento social e afeta as relações e a qualidade de vida das pessoas e da sociedade como um todo. Constitui-se num fenômeno mundial que independente de raça, grupo político, idade, condição socioeconômica, educação, credo ou religião, orientação sexual ou atividade ocupacional (Marzial 2004).

\footnotetext{
1 Universidade Federal do Rio Grande do Sul, Instituto de Psicologia do Desenvolvimento e da Personalidade, Laboratório de Psicologia Experimental, Neurociências e Comportamento. R. Ramiro Barcelos, 2600, Santa Cecília, 90035-003, Porto Alegre, RS, Brasil. Correspondência para/Correspondence to: R.M.M. ALMEIDA. E-mails: <rosa_almeida@yahoo.com>; <rosa.almeida@ufrgs.br>.
} 
O consumo de drogas é um importante facilitador de situações de violência, pois não faltam evidências do envolvimento das drogas nos homicídios, suicídios, violência doméstica, crimes sexuais, atropelamentos e acidentes de trânsito (Laranjeira, Duailibi \& Pinsky, 2005; Moraes, 2001; Tavares, 2008).

A violência e a agressão são consideradas as maiores causas de morte e ferimentos, pois são"problemas primários de saúde pública", acarretando gastos de bilhões de dólares para toda a sociedade (Liu \& Werker, 2005; Siegel, Bhatt, Bhatt \& Zalcman, 2007).

São fatores de risco as desigualdades sociais e o uso abusivo e/ou tráfico de drogas, bem como a disponibilidade de armas de fogo, que ocasionam consequências desastrosas no âmbito social, psicológico, político e econômico (Almeida, 2002; Briceño-León, Villaveces \& Concha-Eastman, 2008; Meneghel, Giugiani \& Falceto, 1998).

Essa revisão teve por objetivo analisar a relação entre violência, dependência química e transtornos mentais na população carcerária masculina.

O presente trabalho realizou uma análise de artigos científicos selecionados nas fontes eletrônicas SciELO, PubMed e Web of Science, no período entre 1998 e 2008. Na elaboração da revisão e na discussão dos dados, foram utilizados os descritores: violência (violence); dependência de drogas (drug dependence), transtornos mentais (mental disturbs) e prisioneiros (prisoners). No total foram encontrados 408 artigos sobre a temática, tendo sido utilizados 60 deles, juntamente com 23 livros e teses que abordam a problemática em questão.

\section{Resultados e Discussão}

A Organização Mundial da Saúde (OMS) (2002) define violência como o uso intencional da força, ou poder, em forma de ameaça contra si mesmo, outra pessoa, grupo ou comunidade, que ocasiona (ou tem grandes probabilidades de ocasionar) lesão, morte, dano psíquico, alterações do desenvolvimento e privações. Também pode ser compreendida como uma ação, sendo considerada um fenômeno natural e civilizatório (Soethe, 2006).
A violência é um comportamento mal adaptativo, envolvendo a agressão que é direcionada inadequadamente a um foco individual ou mesmo coletivo (Bobbio, 1998; Gauer, 2001; Kristensen, Lima, Ferlin, Flores \&Hackmann, 2003). A agressividade é parte integrante e natural do processo evolutivo, pois cumpre o importante papel de garantia da sobrevivência das espécies (Marzial, 2004; Soethe, 2006). Ainda pode ser considerada um comportamento social que está relacionado às atitudes em busca da realização de um objetivo específico, ou às ações direcionadas contra estímulos ameaçadores, com intenção de causar dano, tanto para o ataque como para a defesa. É, também, uma disposição para agredir ou desenvolver condutas hostis e destrutivas, que podem gerar danos à integridade física, moral ou psicológica de outrem, por meio da utilização de força física ou verbal (Corsi, 1997; Kristensen et al., 2003; Niehoff, 1999; Rasia-Filho, Giovenardi \& Almeida, 2008).

Os conceitos de violência, agressão e agressividade envolvem diferentes enfoques e podem estar inseridos dentro da biologia, do estudo do comportamento, da psiquiatria ou de abordagens mais amplas, como da violência estrutural (Maldonado, 1997; Meneghel et al., 1998).

O comportamento violento ou antissocial pode ter suas origens na genética, no funcionamento cerebral alterado e/ou também no meio ambiente. Assim, o comportamento humano deve ser compreendido a partir de uma perspectiva biopsicossocial, em que qualquer transtorno apresentado pelo sujeito deve ser percebido, levando-se em consideração sua história, suas predisposições constitucionais, a etapa de vida que vivência, a presença ou não de doenças associadas e sua relação com a família e o meio social em que está inserido (Gauer, 2001; Raine, 2002).

É preciso reconhecer a existência provável de laços entre determinadas realidades que tendem a conviver, como violência, pobreza, baixa escolaridade, falta de oportunidade de trabalho, angústia, insegurança, depressão, baixa autoestima e alcoolismo. Porém, Santos, Barcellos, Carvalho e Flores (2001) ressaltam que a violência não é um fenômeno exclusivo das diferenças de classes, tornando-se perversa sua associação com a pobreza, pois acaba atingindo as populações mais carentes com uma dupla violência: já punidas pela violência gerada pela própria pobreza, sofrem por serem 
consideradas classes perigosas (Soares, Almeida-Filho, Coutinho \& Mari, 2004).

A violência aparece nas relações humanas através dos comportamentos e ações que as pessoas têm nos seus relacionamentos do dia a dia. Para entender as razões de um comportamento, tem-se que levar em consideração que ele é consequência do movimento de diversos fatores que se interligam, gerando condicionamentos mútuos e complexos (Kusnetzoff, 1982).

Há três formas de desempenhar papéis em atos violentos, segundo Koller (1999): como vítima, como ator ou como testemunha. Mas essa divisão é apenas didática, pois na prática tais formas não são separadas umas das outras e podem ocorrer paralelamente. Segundo a mesma autora, o agressor expressa sua violência pela transgressão de normas de convivência na sociedade, ou pelo rompimento de uma regra moral que protege o patrimônio e a vida.

A origem do comportamento violento é multifatorial, não estando até agora completamente esclarecida. Porém, para que haja um controle dos impulsos agressivos, é necessário que o sistema nervoso esteja sintonizado com os requisitos do meio ambiente, tendo capacidade de avaliar a realidade, por meio de um juízo crítico preservado. Sendo assim, não pode estar comprometido por doenças ou uso de drogas (Gauer, 2001).

As drogas podem ser estímulos, motivos, respostas ou elementos mediadores de comportamentos sociais violentos. As evidências apontam que as drogas apresentam papel relevante nos contextos em que são usadas. Por exemplo, o abuso do álcool pode aumentar a possibilidade de haver comportamento agressivo, porém isso não ocorre de forma generalizada, já que as consequências do uso abusivo do álcool e outras drogas dependem de fatores individuais, sociais e culturais (Borders, Barnwell \& Earleywine, 2007; Laranjeira et al., 2005; Minayo \& Deslandes, 1998).

Cabe ressaltar que nem todo usuário de drogas evoluirá para a dependência, pois a atitude do indivíduo frente à substância varia de acordo com sua história de vida, seu caráter, seus objetivos e dificuldades, bem como de acordo com suas motivações e desejos conscientes e inconscientes (Lourenço, 2001).
A irritabilidade, as frustrações e a carência afetiva presentes na rotina diária podem gerar depressão e ansiedade insuportáveis, que levam a buscar alívio de várias maneiras, entre elas através de atitudes autoagressivas, como o uso abusivo de drogas (Lourenço 2001). O consumo de substâncias psicoativas, com finalidades as mais diversas, existe inserido em todas as culturas desde os primórdios da história. Como fatores desencadeantes do uso das drogas, podem-se citar o desejo de transcendência, a cura de doenças, o alívio da ansiedade, a busca da imortalidade e do prazer, a fuga da dor, a inclusão social e o reconhecimento (Sanceverino \& Abreu, 2004; Moraes, 2001; Tavares, Béria \& Lima, 2001).

A relação drogas-violência é muito complexa, podendo ter suas origens em fatores como personalidade, antecedentes familiares de dependência, fatores genéticos, características de temperamento, relacionamento familiar fragilizado, transtornos de personalidade e todas as circunstâncias sociais que predisporiam ao crime e à dependência química. Porém, em ambientes onde houver maior aceitação da violência e menor receio de suas consequências sociais, físicas e legais, poderá existir um índice maior de criminalidade e de dependência a substâncias psicoativas. Assim, provavelmente, os indivíduos que cometem crimes estariam mais expostos a situações socioculturais em que o uso de drogas pode ser perdoado ou encorajado (Laranjeira et al., 2005; Lavine, 1997; Moraes, 2001)

O uso da cocaína, dos barbitúricos, das anfetaminas e/ou dos esteroides podem motivar atitudes e comportamentos violentos. Os usuários de cocaína têm problemas de supressão das atividades neurotransmissoras, podendo ser vítimas de depressão, paranoia e irritabilidade. Já o abuso de álcool pode acarretar mudanças de personalidade e de comportamento, que prejudicam as interações sociais e pessoais, destacando-se a negligência, a impulsividade, a falta de empatia e controle emocional, sintomas esses que aumentariam o risco de conduta agressiva (Brun \& Andersson, 2001; Laranjeira et al., 2005; Minayo \& Deslandes, 1998; Mitchell, Fields, D’Esposito \& Boettiger, 2005; Monnot, Nixon, Lovallo \& Ross, 2001; Sanceverino \& Abreu, 2004).

O consumo de drogas está presente em grande parte dos atos violentos, sendo que nos casos de estupro e atentado ao pudor sua frequência é de 13\% a 50\%. Existe uma relação entre a violência e a impossibilidade 
de controle, em situações de abuso de droga, que tanto pode ser usada antes como depois do evento violento (Babor et al., 2000; Guareschi, Weber, Comunello \& Nardini, 2006; Minayo \& Deslandes, 1998).

Em 2005, o II Levantamento Domiciliar de Drogas e Álcool, realizado pelo CEBRID, apresentou números alarmantes em relação ao uso de drogas no Brasil, com estimativa de dependentes de álcool em 12,3\% e de tabaco em 10,1\%. O uso da maconha aparece em primeiro lugar entre as drogas ilícitas, sendo uma das mais citadas, com 8,8\% dos entrevistados, seguido pelos solventes (6,1\%) e pela heroína (0,09\%), não havendo relato do consumo de drogas injetáveis. A prevalência do uso de outras drogas foi de 3,8\%. Ainda, segundo a pesquisa, 19,5\% do total de homens entrevistados mostraram dependência do álcool e 9,1\% relataram perda de controle ao beber. A faixa etária na qual aparecem as maiores porcentagens de dependentes (19,3\%) foi de 18 a 24 anos de idade (Carlini, Galduróz, Noto \& Nappo, 2006).

Quanto à distribuição de dependentes entre os gêneros, constatou-se, ainda conforme o Levantamento do CEBRID, que a porcentagem de dependentes do gênero masculino é três vezes maior que do feminino. O gênero masculino esteve mais exposto aos riscos físicos associados à bebida e relatou mais problemas associados ao uso de álcool que o feminino. Apenas 11,4\% dos entrevistados apontaram desejo de parar ou diminuir o consumo de bebidas alcoólicas. Nos estados do Paraná, Santa Catarina e Rio Grande do Sul, o álcool e o tabaco são as drogas mais utilizadas pela população e, excetuadas essas duas drogas, o uso das demais foi de $14,8 \%$. A maconha foi a terceira droga mais usada, com 9,7\% de uso na vida e 1,1\% de dependência, considerando a dependência química conforme os critérios do DSM-IV (Carlini, Galduróz, Noto \& Nappo, 2006).

Entre os principais problemas de saúde pública no Brasil, o mais grave é o consumo de álcool, por sua relação com a violência e por ser fator determinante de mais de 10\% de toda a morbidade e mortalidade ocorrida no país. As drogas e o álcool degradam física e moralmente os indivíduos. Quanto aos prejuízos sociais, perpassam por perdas de ordem material, aumento da insegurança e da violência, bem como deterioração do relacionamento humano (Laranjeira \& Romano, 2004;

548 Minayo \& Deslandes, 1998; Serrat, 2001).
A relação entre comportamento violento e transtornos mentais vem sendo analisada de forma cada vez mais criteriosa. Porém, ainda não há comprovação científica da existência de associação entre violência e transtornos mentais (Abdalla-Filho 2004). Conforme Webster e Bailes (2000), houve um grande exagero, nos anos 60, referente à predisposição de periculosidade nos indivíduos que apresentavam transtornos mentais (Gostin, 2008).

Há grande probabilidade de se encontrar um número significativo de presidiários portadores de transtornos mentais que não estão recebendo acompanhamento adequado, embora anteriormente já tenham feito algum tratamento para esquizofrenia, transtorno bipolar, depressão ou ansiedade (Fonseca, 2006; Blitz, Wolff \& Shi, 2008).

Os transtornos mentais são alterações do funcionamento cerebral que interferem no desempenho familiar, social e na capacidade de autocrítica, tolerância e possibilidade de ter prazer na vida em geral. Estudos com sociopatas e criminosos constataram o diagnóstico de personalidade antissocial e outros transtornos de personalidade (Gauer, 2001; Raine, 2002). O comportamento antissocial é considerado o mais abrangente, por estar associado a violência, suicídio e comportamento de risco; refere-se a condutas condenadas pela sociedade, com ou sem a transgressão às leis do Estado (Abdalla-Filho, 2004; Guy, Poythress, Douglas, Skeem \& Edens, 2008).

Apesar das várias pesquisas sobre o funcionamento cerebral diferenciado encontrado em sociopatas, ressalta-se o cuidado em não estigmatizar sujeitos que apresentam padrões de ativação cerebral diferentes. 0 que deve ser proposto são intervenções precoces, capazes de evitar situações extremas de violência, que podem ocorrer quando os aspectos genéticos, sociais e neurofuncionais se juntam (Raine, 2002).

O transtorno psiquiátrico, se avaliado de forma isolada, é um critério pobre de avaliação de risco, pois deve-se considerar também a presença de outros fatores biopsicossociais que representam riscos significativos, como o uso abusivo de drogas, a inexistência de vínculos sociais e familiares e a não-aderência a tratamento psiquiátrico (Abdalla-Filho, 2004; Harris \& Rice, 1997; Thomson, 1999). 
É necessário investir em pesquisas mais aprofundadas, que adotem uma postura equilibrada no sentido de não supervalorizar nem tampouco negligenciar a relação dos transtornos mentais ao comportamento violento. Nos casos de transtorno mental, a sociedade não pode julgar o preso como único responsável pela ação violenta, em razão da ausência de autocrítica e da incapacidade de compreender o caráter criminoso de sua atitude. Entre os transtornos mentais, considera-se importante abordar também a depressão e a ansiedade vinculadas ao comportamento violento, tendo em vista a restrita literatura científica brasileira sobre o tema (Abdalla-Filho, 2004; Dallari, 2007).

A impulsividade e os transtornos emocionais, muitas vezes, acompanham atos violentos, sendo que indivíduos com diagnóstico pregresso de transtorno de ansiedade e depressão são considerados de três a quatro vezes mais propensos a apresentar comportamento violento do que aquelas sem distúrbios (Badawy, 2003; Corrigan \& Watson, 2005).

Bandura (1997), seguindo a abordagem cognitiva, ressalta que a depressão é causada por três fatores principais: ambientais, cognitivos e comportamentais. Os sintomas depressivos podem se manifestar de várias formas, principalmente com modificações de humor e alterações motoras, somáticas, sociais, emocionais e cognitivas. As alterações de humor podem ser: tristeza, perda de interesse por qualquer atividade, falta de prazer, crises de choro, irritabilidade. As alterações motoras incluem inibição ou retardo dos movimentos e agitação. As mudanças somáticas aparecem como alterações no sono, variações do apetite e peso, perda de libido e fadiga. As alterações sociais incluem apatia, isolamento e incapacidade para o desempenho de tarefas cotidianas. As alterações emocionais relacionam-se com desesperança, desamparo, ideia de culpa e de suicídio, indecisão e perda de insight, isto é, do reconhecimento da doença. Já as alterações cognitivas... (desenvolver, já que elas foram anunciadas acima). A depressão pode ainda ser mascarada por sintomas como dependência ou uso abusivo de drogas, obesidade ou agitação psicomotora (Rezende \& Derchain, 2005; Soares et al., 2004).

Os comportamentos antissociais e violentos são comuns na "depressão mascarada" e podem ser considerados como grandes fatores de risco para o suicídio. Frequentemente, sintomas como a irritabilidade e a intolerância acompanham o quadro depressivo. Nesses casos, podem surgir sentimentos de culpa no agressor, porém, devido à impulsividade característica desse transtorno, há grande dificuldade em conseguir o controle de seus gestos e atitudes em situações que lhe despertem raiva (Conner, Cox, Tian, Nisbet \& Conwell, 2001; Rezende \& Derchain, 2005; Soares et al., 2004).

Há uma interação recíproca que mantém o transtorno depressivo, sendo esse círculo vicioso movido pela retração do sujeito depressivo, que influencia o comportamento das pessoas de seu convívio, que passam a excluí-lo, realimentando sua retração e autorrejeição (Beck, Rush, Shaw \& Emery, 1997; Rutter, 1995).

Cabe ressaltar que o aparecimento de alguns sintomas depressivos não significa necessariamente que se trata de uma disfunção psicológica, pois a depressão pode ser compreendida como um contínuo, podendo variar desde uma resposta adaptativa até a incapacitação e ideação suicida. Assim, os sintomas depressivos são respostas normais ao stress cotidiano, sendo psicopatológicos somente quando se apresentam de forma desproporcional ao evento causador e por um período considerável de tempo (Soethe, 2006; Beck, Steer \& Garbin, 1988).

Entre os transtornos psiquiátricos mais frequentes na população em geral, encontra-se, além da depressão, a ansiedade, com prevalência de 12,5\% a 15\% ao longo da vida (Castillo \& Recondo, 2000). Além de muito frequentes, os sintomas ansiosos estão entre os mais comuns, podendo ser encontrados em qualquer pessoa em determinados períodos de sua vida (Andrade \& Gorenstein, 2001).

A ansiedade é considerada um estado emocional que parte da experiência subjetiva de medo, ou de uma emoção relacionada, como terror e pânico. É uma emoção desagradável, podendo ser uma sensação de morte ou colapso iminente, e está sempre direcionada ao futuro. Porém, não há necessariamente um risco real e, quando ele existe, a emoção é intensamente desproporcional ao fato. Os desconfortos físicos principais durante o estado de ansiedade são aperto no peito, na garganta, dificuldade para respirar e fraqueza nas pernas (Andrade \& Gorenstein, 2001).

O transtorno de ansiedade apresenta componentes psicológicos e fisiológicos que fazem parte das 
emoções humanas normais, configurando-se como impulsor do desenvolvimento. Ele se torna patológico quando é desproporcional à situação que o desencadeia, ou quando não existe um objeto específico ao qual se relacione (Andrade \& Gorestein, 2001; Biaggio, 1998). A ansiedade é um sentimento de medo e apreensão, decorrente da antecipação de perigo de algo desconhecido ou estranho que gera tensão ou desconforto.

A ansiedade e a raiva estão associadas com sobreposição e padrões distintos de atividade cerebral. A maneira prática de se diferenciar ansiedade normal de ansiedade patológica é basicamente avaliar se a reação ansiosa é de curta duração, autolimitada e relacionada ao estímulo do momento, ou não (Amen, Stubblefield, Camicheal \& Thisted, 1999; Castillo \& Recondo, 2000).

Ao apresentar comportamento violento, o homem demonstra também ansiedade intensa e irritabilidade. Indivíduos agressivos, geralmente, experimentam dificuldades em controlar os impulsos emocionais, o que prejudica sua relação social e familiar. Porém, a ocorrência de agressão depende de fatores individuais, bem como do contexto social em que o sujeito se encontra. A ocorrência de experiências estressoras e ansiosas potencializa a diminuição da autoestima e o surgimento de sentimentos de desamparo e rejeição. A autoestima elevada tem um impacto positivo no bem-estar, na qualidade de vida e, principalmente, na maneira de resolver conflitos (Andrade \& Gorenstein, 2001; Badawy, 2003; Coopersmith, 1989).

\section{Considerações Finais}

A relação entre o consumo de álcool ou outras drogas e o comportamento violento é um fenômeno complexo, que vem sendo estudado por diferentes abordagens acadêmicas e desafia pesquisadores e formuladores de políticas na área de segurança pública. Há constatação da alta proporção de atos violentos, quando o álcool ou as drogas ilícitas estão presentes entre agressores, suas vítimas ou em ambos. Por outro lado, o processo de redemocratização do Estado brasileiro não foi suficientemente acompanhado de medidas que reduzissem o elevado índice de exclusão social, o que contribui para que haja cada vez mais internos em instituições carcerárias (Chermack \& Giancola, 1997;

550 Chalub \& Telles, 2006; Oliveira, 2003).
Dificilmente os homens se consideram agressores e, mesmo quando alguém os reconhece como tal, a violência em seu cotidiano continua sendo tratada de forma irrelevante. Conforme Hamberger e Patel (2001), características isoladas já foram descritas a respeito de homens agressores, tais como impulsividade, isolamento social, ciúme, abuso/dependência de substâncias psicoativas e baixa autoestima. A dependência do álcool ou de outras drogas encontra-se, muitas vezes, em comorbidade com outros transtornos, destacando-se ansiedade, depressão e personalidade antissocial. Essa visão possibilita novas formas de acompanhamento, bem como o direcionamento a tópicos que envolvam desde o controle da raiva até o tratamento da dependência química (Enoch, 2003; Guareschi et al., 2006).

O estudo das características emocionais de homens agressores e do contexto social no qual estão inseridos é de grande relevância para se obter sucesso no rompimento do ciclo de violência. Apenas com a reclusão, sem o adequado acompanhamento, avaliação e tratamento, as chances de o sujeito reingressar na instituição do sistema prisional são 80\% maiores (Gonzaga, Santos \& Bacaarin, 2002). A punição não gera transformação se não houver investimento também nos cuidados com todos os demais aspectos envolvidos nas situações de violência na contemporaneidade (Gonzaga, Santos \& Bacaarin, 2002; Parker \& Auerhaln, 1998; Patto, 2000).

Embora se acredite que o comportamento violento possa ser modificado, de acordo com Gauer (2001), é imprescindível que todos os fatores envolvidos também sofram transformações. Atuar no âmbito biopsicossocial de forma conjunta e interdisciplinar impede que um dos fatores continue desviando o comportamento para atos violentos. Assim, tornam-se de grande relevância as iniciativas de pesquisas nessa área, que sejam direcionadas ao conhecimento do homem preso, como forma de viabilizar sua reinserção social, no intuito de diminuir os índices de reingresso no sistema penitenciário.

\section{Referências}

Abdalla-Filho, E. (2004). Avaliação de risco de violência em psiquiatria forense. Revista de Psiquiatria Clínica, 31 (6), 279-284 
Almeida, S. (2002). Violência e subjetividade. In C. Rauter, E. Passos \& R. Benevides-de-Barros (Eds.), Clínica e política: subjetividade e violação dos direitos humanos (pp.45-50). Rio de Janeiro: TeCorá.

Amen, D., Stubblefield, M., Camicheal, B., \& Thisted, R. (1999). Brain SPECT findings and aggressiveness. Annual Clinical Psychiatry, 8 (3), 129-37.

Andrade, L., \& Gorenstein C. (2001). Aspectos gerais das esca-las de avaliação de ansiedade. Revista de Psiquiatria Clínica, 25 (6), 285-290.

Babor, T. F., Caetano R., Caswell S., Edwards G., Giesbrech N., Grahan K., et al. (2003). Alcohol: no ordinary commodity: research and public policy (pp.57-92). Oxford: Oxford University Press.

Badawy, A. (2003). Alcohol and violence and the possible role of serotonin. Criminal Behavior Mental Health, 13 (1), $31-44$.

Bandura, A. (1997). Self-efficacy: the exercise of control. New York: W. H. Freeman and Company.

Beck, A., Steer, R., \& Garbin, M. (1988). Psychometric properties of the beck depression inventory: twenty-five years of evaluation. Clinical Psychology Review, 8, 77-100.

Beck, A., Rush, A., Shaw, B., \& Emery, G. (1997). Terapia cognitiva da depressão. Porto Alegre: Artes Médicas.

Biaggio. A. (1998). Ansiedade, raiva e depressão na concepção de C. D. Spielberger. Revista Psiquiatria e Clínica, 25 (6 Edição Especial), 291-293.

Blitz, C.; Wolff, N., \& Shi, J. (2008). Physical victimization in prison: the role of menal illness. International of Journal Law Psychiatry, 31 (5), 385-393.

Bobbio, N. (1998). Dicionário de política (Vol. 2, $11^{\text {a ed.). Bra- }}$ sília: Universidade de Brasília.

Borders, A., Barnwell, S., \& Earleywine, M. (2007). Alcoholagression expectancies and dispositional rumination moderate the effect of alcohol consumption on alcoholrelated Aggression and hostility. Aggressive Begavior, 33 (4), 327-338.

Briceño-León, R., Villaveces, A., \& Concha-Eastman, A. (2008). Understanding the uneven distribution of the incidence of homicide in Latin America. International Journal of Epidemiology, 37 (4), 751-7.

Brun, A., \& Andersson, J. (2001). Frontal dysfunction and frontal cortical synapse loss in alcoholism-the main cause of alcohol dementia? Dementia Geriatric Cognitive Disorder, 12 (4), 289-294.

Carlini E., Galduróz J., Noto A., \& Nappo S. (2002). I Levantamento domiciliar sobre o uso de drogas no Brasil: estudo envolvendo as 107 maiores cidades do país - 2001. São Paulo: Unifesp.

Castillo, A., \& Recondo R. (2000). Transtornos de ansiedade. Revista Brasileira de Psiquiatria, 22 (Supl. 2), 20-23.

Conner, K., Cox, C., Tian, L., Nisbet, P., \& Conwell, Y. (2001). Violence, alcohol, and completed suicide: a case-control study. American Journal of Psychiatry, 158 (10), 1701-1705.

Coopersmith, S. (1989). Coopersmith self-esteem inventory. Palo Alto, CA: Consulting Psychologists Press.
Corrigan, P., \& Watson, A. (2005). Findings from the National Comorbidity Survey on the frequency of violent behavior in individuals with psychiatric disorders. Psychiatry Research, 136 (2-3), 153-162.

Corsi, J. (1997). Violência familiar: uma mirada interdiscipinaria sobre um grave problema social (3a ed.). Buenos Aires: Paidos.

Dallari, S. (2007). Direitos das pessoas com transtorno mental autoras de delitos. Cadernos de Saúde Pública, 23 (9), 1995-2012.

Del Prette, Z., \& Del Prette, A. (1999). Psicologia das habilidades sociais: terapia eeducação (2a ed.). Petrópolis: Vozes.

Enoch, M. (2003). Pharmacogenomics of alchol respnse and addiction. American Journal of Pharmacogenomics, 3 (4), 217-232.

Fonseca K. (2006). (Re)Pensando o crime como uma relação de antagonismo entre seus autores e a sociedade. Psicologia: Ciência \& Profissão, 26 (4), 532-547.

Gauer, G. (2001). Personalidade e conduta violenta. Civitas: Revista de Ciências Sociais, 1 (2), 45-65.

Gonzaga, M., Santos H., \& Bacaarin. (2002). A cidadania por um fio: a luta pela inclusão dos apenados na sociedade. Maringá: Dental Press.

Gostin, L. (2008). "Old" and "new" instituitions for persons with mental illness: treatment, punishment or preventive confinement? Health, 122 (9), 906-913.

Guareschi, N., Weber, A., Comunello, L., \& Nardini, M. (2006). Discussões sobre violência: trabalhando a produção de sentidos. Psicologia Reflexão Critica, 19 (1), 122-130

Guy, L., Poythress, N., Douglas, K., Skeem, J., \& Edens, J. (2008). Correspondence between serf-report and interview-based assessments of antisocial personality disorder. Psychological Assesse, 20 (1), 47-54.

Hamberger, L. K., \& Patel, D. (2001). Domestic violence assessments and prevention. Clinics in Family Pratice, 3 (1), 93-111.

Harris, G., \& Rice, M. (1997). Risk appraisal and management of violent behavior. Psychiatric Services, 48 (9),1168-75.

Instituto Brasileiro de Geografia e Estatística. Recuperado em janeiro 10, 2008, disponível em: http://www.ibge. gov.br

Koller, S. (1999). Violência doméstica: uma visão ecológica. In AMENCAR. (Org.), Violência doméstica (pp.32-42). Brasília: Unicef.

Kristensen, C., Lima, J., Ferlin, M., Flores, R., \& Hackmann, P. (2003). Fatores etiológicos da agressão física: uma revisão teórica. Estudos de Psicologia, 8 (1), 175-184.

Kusnetzoff, R. (1982). As áreas do saber. São Paulo: Cortez.

Laranjeira, R., Duailibi, S., \& Pinsky, I. (2005). Álcool e violência: a psiquiatria e a saúde pública. Revista Brasileira de Psiquiatria, 27 (3), 176-177.

Laranjeira, R., \& Romano, M. (2004). Brazilian consensus on public policies on alcohol. Revista Brasileira de Psiquiatria, 26 (1), 68-77. 
Lavine, R. (1997). Psychopharmacological treatment of aggression and violence in the substance using population. Journal of Psychoactive Drugs, 29 (4), 321-329.

Liu, J., \& Werker, A. (2005). Biosocial bases of aggressive and violent behavior: implications for nursing studies. International Journal of Nursing Studies, 42 (2), 229-241.

Lourenço, R. (2001). Drogas e álcool prevenção e tratamento. São Paulo: Komedi.

Maldonado, M. (1997). Os construtores da paz: caminhos da prevenção da violência. São Paulo: Moderna.

Marzial M. (2004). A violência no setor saúde. Revista Latino-americana de Enfermagem, 12 (2), 147-148.

Meneghel, S., Giugliani, E., \& Falceto, O. (1998). Relações entre violência doméstica e agressividade na adolescência. Cadernos de Saúde Pública, 14 (2), 327-335.

Minayo, M. C., \& Deslandes, F. (1998). Drogas e mudanças bio-psico-sociais que possibilitam a violência: por um enfoque mais amplo das condutas individuais. Cadernos de Saúde Pública, 14 (1), 35-42

Mitchell, J., Fields, H., D’Esposito, M., \& Boettiger, C. (2005). Impulsive responding in alcoholics. Alcoholism: Clinical \& Experimental Research, 29 (12), 2158-2169.

Monnot, M., Nixon, S., Lovallo, W., \& Ross, E. (2001). Altered emotional perception in alcoholics: deficits in affective prosody comprehension. Alcoholism: Clinical and Experimental Research, 25 (3), 362-369.

Moraes, R. (2001). Drogas e álcool prevenção e tratamento. São Paulo: Komedi.

Niehoff, D. (1999). The biology of violence. Nova York: Free Press.

Oliveira, A. (2003). A violência e a criminalidade como entraves à Democratização da sociedade brasileira. Caderno $C R H, 38,239-265$

Organização Mundial da Saúde. (2002). Informe mundial sobre la violencia y salud. Genebra: Autor.

Patto, M. (2000). Mutações do cativeiro: escrito de psicologia e política. São Paulo: Edusp.

Raine, A (2002). Biosocial studies of anti-social and violent behavior in children and adults: a review. Journal of Abnormgl Child Psychology, 30, 311-326.

Rasia-Filho, A., Giovenardi, M., \& Almeida, R. (2008). Drugs and aggression. Recent Patents CNS Drug Discov, 3 (1), 40-9.
Rezende, V., \& Derchain, S. (2005). Depressão e ansiedade nos cuidadores de mulheres em fase terminal de câncer de mama e ginecológico. Revista Brasileira Ginecologia e Obstetricia, 27 (12), 737-743

Rutter, M. (1995). Clinical implications of attachment concepts: Retrospect and prospect. Journal of Child Psychology and Psychiatry, 36, 549-571.

Sanceverino, S. \& Abreu, J. (2004). Aspectos epidemiológicos do uso de drogas entre estudantes do ensino médio no município de Palhoça. Ciência \& Saúde Coletiva, 9 (4), 1047-1056.

Santos, S., Barcellos, C., Carvalho, M., \& Flores, R. (2001). Detecção de aglomerados espaciais de óbitos por causas violentas em Porto Alegre. Rio Grande do Sul, Brasil, 1996. Cadernos de Saúde Pública, 17 (5), 1141-1151.

Serrat, S. (2001). Drogas e álcool prevenção e tratamento. São Paulo: Komedi.

Siegel, A., Bhatt, S., Bhatt, R., \& Zalcman, S. (2007). The neurobiological bases for development of pharmacological traeatments of aggressive disorders. Current Neuropharmacol, 5 (2), 135-47.

Soares, K., Almeida-Filho, N., Coutinho, E., \& Mari, J. (2004). Sintomas depressivos entre os adolescentes e adultos de uma amostra populacional de três centros urbanos brasileiros: análise dos dados do Estudo Multicêntrico de Morbidade Psiquiátrica. Revista de Psiquiatria Clínica, 26 (5), 218-224

Soethe, J. (2006). Conexões para uma nova civilização. São Leopoldo: Oikos.

Tavares, F. (2008). Das Lágrimas à esperança: o processo de fortalecimento das mulheres em situação de violência doméstica. Disponível em http://tede.pucrs.br/tde_ busca/arquivo.php?codArquivo=1378

Tavares, B., Béria, J., \& Lima, M. (2001). Prevalência do uso de drogas e desempenho escolar entre adolescentes. Revista de Saúde Pública, 35 (2), 150-158.

Thomson, L. (1999). Clinical management in forensic psychiatry. Journal of Forensic Psychiatry, 10 (2), 367-90.

Webster, C., \& Bailes, G. (2000). Assessing violence risk in mentally and personality disordered individuals. In C.R. Hollin (Ed.), Handbook of offender assessment and treatment (pp.71-84). New York: John Wiley \& Sons.

Recebido em: 12/11/2008

Versão final reapresentada em: 14/7/2010

Aprovado em: 18/8/2010 